\title{
A systematic survey on reporting and methods for handling missing participant data for continuous outcomes in randomized controlled trials
}

\author{
Yuqing Zhanga, ${ }^{\mathrm{a} b}$, Ivan D. Flórez ${ }^{\mathrm{a}, \mathrm{c}}$, Luis E. Colunga Lozano ${ }^{\mathrm{d}}$, Fazila Abu Bakar Aloweni ${ }^{\mathrm{e}}$, \\ Sean Alexander Kennedy ${ }^{\mathrm{f}}$, Aihua $\mathrm{Li}^{\mathrm{a}}$, Samantha Craigie ${ }^{\mathrm{g}}$, Shiyuan Zhang ${ }^{\mathrm{h}}$, Arnav Agarwal ${ }^{\mathrm{i}}$, \\ Luciane C. Lopes ${ }^{\mathrm{j}, \mathrm{k}}$, Tahira Devji ${ }^{\mathrm{a}}$, Wojtek Wiercioch ${ }^{\mathrm{a}}$, John J. Riva, ${ }^{\mathrm{a}, \mathrm{l}}$, Mengxiao Wang ${ }^{\mathrm{a}}$, \\ Xuejing Jin ${ }^{\mathrm{a}}$, Yutong Fei ${ }^{\mathrm{a}, \mathrm{m}}$, Paul Alexander ${ }^{\mathrm{a}}$, Gian Paolo Morgano, ${ }^{\mathrm{a}}$, Yuan Zhang ${ }^{\mathrm{a}}$, \\ Alonso Carrasco-Labra ${ }^{\mathrm{a}, \mathrm{n}}$, Lara A. Kahale ${ }^{\mathrm{o}}$, Elie A. Akl ${ }^{\mathrm{a}, \mathrm{o}}$, Holger J. Schünemann ${ }^{\mathrm{p}}$, \\ Lehana Thabane ${ }^{\mathrm{a}}$, Gordon H. Guyatt ${ }^{\mathrm{p}, *}$ \\ a Department of Health Research Methods, Evidence, and Impact, McMaster University, Hamilton, Ontario, Canada \\ ${ }^{\mathrm{b}}$ Guang'anmen Hospital China Academy of Chinese Medical Science, Xicheng District, Beijing, China \\ ${ }^{\mathrm{c}}$ Department of Pediatrics, University of Antioquia, Medellín, Colombia \\ ${ }^{\mathrm{d}}$ Department of Critical Care, Hospital Angeles del Carmen, Guadalajara, Jalisco, Mexico \\ ${ }^{\mathrm{e}}$ Nursing Division, Singapore General Hospital, Singapore \\ ${ }^{\mathrm{f}}$ Department of Diagnostic Radiology, University of Toronto, Toronto, Ontario, Canada \\ ${ }^{\mathrm{g}}$ Department of Anesthesiology, Michael G. DeGroote National Pain Centre, Hamilton, Ontario, Canada \\ ${ }^{\mathrm{h}}$ Health Economics and Outcomes Research, Medical Affairs, GSK, Mississauga, Ontario, Canada \\ ${ }^{\mathrm{i}}$ Faculty of Medicine, University of Toronto, Toronto, Ontario, Canada \\ ${ }^{\mathrm{j} D e p a r t m e n t ~ o f ~ P h a r m a c e u t i c a l ~ s c i e n c e, ~ U n i v e r s i d a d e ~ d e ~ S o r o c a b a, ~ S a ̃ o ~ P a u l o, ~ B r a z i l ~}$ \\ ${ }^{\mathrm{k}}$ Department of Pharmaceutical science, Universidade Estadual Paulista “Julio de Mesquita Filho”, São Paulo, Brazil \\ ${ }^{1}$ Department of Family Medicine, McMaster University, Hamilton, Ontario, Canada \\ ${ }^{\mathrm{m}}$ Center for Evidence-Based Chinese Medicine, Beijing University of Chinese Medicine, Chaoyang Qu, China \\ ${ }^{\mathrm{n}}$ Evidence based dentistry unit and Department of oral and maxillo-facial surgery, Universidad de Chile, Santiago, Región Metropolitana, Chile \\ ${ }^{\circ}$ Department of Internal Medicine, American University of Beirut, Riad El-Solh, Beirut, Lebanon \\ ${ }^{\mathrm{p}}$ Department of Medicine and Department of Health Research Methods, Evidence, and Impact, Hamilton, Ontario, Canada
}

Accepted 24 May 2017; Published online 3 June 2017

\begin{abstract}
Objective: To assess analytic approaches randomized controlled trial (RCT) authors use to address missing participant data (MPD) for patient-important continuous outcomes.

Study Design and Setting: We conducted a systematic survey of RCTs published in 2014 in the core clinical journals that reported at least one patient-important outcome analyzed as a continuous variable.

Results: Among 200 studies, 187 (93.5\%) trials explicitly reported whether MPD occurred. In the 163 (81.5\%) trials that reported the occurrence of MPD, the median and interquartile ranges of the percentage of participants with MPD were $11.4 \%(2.5 \%-22.6 \%)$. Among the 147 trials in which authors made clear their analytical approach to MPD, the approaches chosen included available data only (109, 67\%); mixed-effect models (10, 6.1\%); multiple imputation (9, 4.5\%); and last observation carried forward (9, 4.5). Of the 163 studies reporting MPD, 16 $(9.8 \%)$ conducted sensitivity analyses examining the impact of the MPD and (18, 11.1\%) discussed the risk of bias associated with MPD.

Conclusion: RCTs reporting continuous outcomes typically have over $10 \%$ of participant data missing. Most RCTs failed to use optimal analytic methods, and very few conducted sensitivity analyses addressing the possible impact of MPD or commented on how MPD might influence risk of bias. (c) 2017 Elsevier Inc. All rights reserved.
\end{abstract}

Keywords: Missing participant data; Continuous outcome; Analytic approaches; MPD; Randomized controlled trials; Lost to follow-up

\footnotetext{
Funding: None.

Conflict of interest: None.

* Corresponding author. Tel.: (905) 525 9140x22900; fax: (905) 524

E-mail address: guyatt@mcmaster.ca (G.H. Guyatt).
} 3841.

\section{Introduction}

Missing participant data (MPD) in randomized controlled trials (RCTs) - also referred to loss to follow-up, discontinued prematurely, or outcome not assessable [1]-refers 


\section{What is new?}

\section{Key findings}

- Frequently (over 15\%) trials authors did not state the analysis strategy for MPD; less than $10 \%$ trials conducted sensitivity analyses examining the impact of MPD.

\section{What this adds to what was known?}

- Among the studies that do not use complete case for primary analysis, trialists often used last observation carried forward to deal with MPD, a demonstrably poor analytical approach.

\section{What is the implication and what should change now?}

- When deal with missing continuous data in randomized trials, trialists should use optimal analytic strategies and conduct sensitivity analyses to assess the impact of MPD on risk of bias.

to missing information on outcomes of interest [2]. Although analyzing patients in the groups to which they were randomized will avoid bias for patients with complete data [3-5], it does not address bias due to MPD, which, if it is substantial and the reasons for MPD differ between the intervention and control groups, is likely to bias the results. For instance, if patients destined to experience poorer quality of life at study termination withdraw consent more frequently from the intervention group than from the control group, and are excluded from the analysis, the results will be biased in favor of the treatment.

A common classification of the reason for missing data (also called missing mechanism) includes missing completely at random (MCAR), missing at random (MAR), and not missing at random (NMAR) [6]. When outcome data are MCAR, it indicates no systematic differences between missing and observed values implying that including only those with available data (complete case) in the analysis will not bias point estimates but enlarge the standard error. Outcome data MAR denotes an explainable systematic difference between missing and observed values based on observed data. Ignoring missing data may cause bias in this case and imputation or data augmentation methods may reduce the extent of bias.

When outcome data are NMAR, systematic differences between missing and observed values can only be explained by unobserved data (eg, a person not responding to treatment is more likely not to provide an observation) [7]. NMAR requires conducting sensitivity analysis comparing effect estimates under different missing mechanisms $[6,8]$. Seldom if ever can investigators be confident that their data are MCAR; thus, assuming some degree of MAR or NMAR is likely to be a more appropriate approach.
Despite the fact that investigators often expend enormous effort to prevent MPD, as the previous series (paper 1) mentioned, MPD is frequent in RCTs across all therapeutic areas [9-12].

Researchers have thoroughly investigated how RCT authors have dealt with MPD in studies focusing on dichotomous outcomes $[1,12,13]$. Dealing with continuous MPD has special challenges [14]. Considering the serious threat of bias from MPD, statisticians and methodologists have developed a variety of methods to deal with MPD in RCTs focusing on continuous outcomes [15-20]. Whether trialists are planning and applying the optimal approaches to handle continuous MPD is unknown.

We therefore conducted a systematic survey of RCTs reporting on continuous outcomes to assess (1) how trial authors report MPD for patient-important continuous outcomes and (2) the analytic approaches they use to address MPD.

\section{Methods}

\subsection{Definitions}

We defined MPD as unavailable data from trial participants that, if available, would have been included in the analysis of the specific outcome in RCTs. We defined a patientimportant outcome as an outcome for which a patient would say "yes" to the following question: "If this outcome were the only thing to change with treatment, would the patient consider receiving this treatment if it is associated with burden, side effects, or cost?" [13]. We used a taxonomy characterizing a hierarchy of the importance of outcomes to select one outcome of primary interest from each trial (Appendix A at www.jclinepi.com). Patient-important continuous outcomes high on this hierarchy include quality of life, symptoms, and functional status. We did not consider surrogate outcomes as patient-important outcomes.

We defined complete case analysis as excluding all patients with any missing value for the outcome being analyzed [21]. In contrast to the complete case analysis, all available data analyses refer to using all available observations for a particular outcome; this means including data from patients with some missing values for that outcome. All available data analyses are commonly seen in trials with repeated measures [2].

\subsection{Eligibility criteria}

\subsubsection{Inclusion criteria}

Eligible studies fulfilled all of the following criteria:

- Published in 2014 in one of 119 core clinical journals;

- Described by authors as an RCT;

- Reported an analysis of data for at least one patientimportant outcome analyzed as a continuous variable.

\subsubsection{Exclusion criteria}

We excluded studies meeting any of the following criteria: 
- RCT reporting time to event outcomes and analyzing those as continuous data;

- Nonhuman trials;

- Cluster RCT, factorial RCT, crossover RCT, n-of-1 trials, cost-utility studies;

- Studies reporting continuous outcomes but analyzed as dichotomous data;

- Meta-analysis of two or more previously published RCTs;

- Secondary analysis of RCTs.

\subsection{Literature search}

We conducted the search using the Cochrane Collaboration's highly sensitive search strategy to identify RCTs through Medline (OVID interface) in the 119 core clinical English journals indexed under Abridged Index Medicus by the National Library of Medicine (available at http://www. nlm.nih.gov/bsd/aim.html) (see Appendix B at www. jclinepi.com).

\subsection{Random sampling of citations}

We retrieved a random sample of the identified citations using generated random numbers from an Excel sheet and retrieved correspondingly citation numbers. We repeatedly sampled and screened identified citations meeting eligibility criteria until we achieved the target sample size.

\subsection{Study selection and data collection}

A team of 20 reviewers, with health research methodology training, worked in pairs using standardized forms to conduct screening of title and abstract, screening of full text, and data abstraction, all independently and in duplicate. We applied a calibration process before screening and data abstraction to ensure accuracy. For both screening and data abstraction, reviewers resolved disagreement through discussion and with the assistance from an independent arbitrator (Y.Z.) if needed. We also reviewed supplementary documents published by the authors to abstract information on detailed description on the reporting and analysis of MPD. We conducted screening and data abstraction using Web-based systematic review software DistillerSR created by Evidence partners (@ 2017 Systematic Review and Literature Review Software from Evidence Partners, https://systematic-review.ca).

\subsection{Selection of outcome and comparison}

For RCTs including more than one patient-important continuous outcome, we selected the primary outcome as the authors reported. If authors reported more than one primary continuous outcome, we selected the first one reported in the abstract. For RCTs including more than one patientimportant continuous outcome with none reported as the primary outcome, we selected the outcome first reported in the abstract, or in the results if not presented in the abstract.
In multiple-arm RCTs, we considered the first comparison reported in the results. For RCTs with multiple followup times, we used the analysis that included all time points or, if there was no such analysis, the analysis focused on the longest follow-up time.

\subsection{Data abstraction}

For each trial, we abstracted data regarding general characteristics, methodological characteristics, reporting, and conducted analytic approach regarding MPD, and the extent of MPD. We recorded the categories trial investigators used to describe participants with potential MPD including ineligible participants, mistakenly randomized, did not receive any intervention, withdrew consent, dead, experienced adverse events, noncompliant or nonadherent, discontinued prematurely, excluded as part of center exclusion, and outcome not assessable. We also recorded the missing mechanism the trial assumed when dealing with MPD, whether authors reported a justification of their approach to MPD, as well as whether trialist assessed risk of bias associated with MPD.

\subsection{Sample size}

We chose a sample size to achieve a precise confidence interval $( \pm 0.05)$ around the proportion of RCTs that conducted primary analytical approach regarding MPD. In the most conservative situation in which the proportion is 0.5 , we would need 200 RCTs to achieve the desired confidence interval $(0.45,0.55)$.

\subsection{Analysis}

We assessed agreement for eligibility between reviewers at both the title and abstract screening stage and the fulltext screening using kappa statistics. We followed the interpretation guideline from Landis and Koch [22]: kappa values of $0-0.20$ represent slight agreement, $0.21-0.40$ fair agreement, $0.41-0.60$ moderate agreement, $0.61-0.80$ substantial agreement, and greater than 0.80 almost perfect agreement.

For all descriptive analyses, we used absolute number and percentages for dichotomous (categorical) variables and mean with standard deviation for continuous outcomes when distribution was normal or near normal. When the distribution was skewed to a large extent, we used median and interquartile range.

\subsubsection{Categories of trial participant investigators considered as having MPD}

For all the categories that trial investigators used to describe participants with potential MPD, such as "ineligible participants," "withdrew consent," "outcome not assessable," we reported the number and percentage of trials documenting the categories. 


\subsubsection{Reporting and extent of MPD}

We calculated the percentage of participants with MPD in each trial and the median and interquartile range of the percentage across all trials. For trials with multiple follow-up times, in addition to these analyses, we also calculated:

- The percentage of missing data points overall through the entire follow-up counted as the number of missing data points divided by total number of possible data points.

- At the last follow-up time, the percentage of missing data points counted as the number of missing data points divided by the total number of possible data points.

We planned to conduct a logistic regression in which the dependent variable was whether trials did or did not report MPD and the independent variables were as follows:

- Sample size

- Type of intervention (pharmaceutical vs. surgical/ invasive nonsurgical vs. others)

- Type of funding (for profit vs. not for profit vs. no funding reported)

- Journal type (top 5 vs. nontop 5)

Top 5 refers to the five general medical journals with the highest impact factor in 2015: Annals of Internal Medicine, British Medical Journal, Journal of the American Medical Association, The Lancet, and New England Journal of Medicine (http://impactfactor.weebly.com/medicine.html).

- Allocation concealment (inadequate vs. adequate)

Our a priori hypotheses were as follows: trials with smaller sample size, for-profit type of funding, nonpharmaceutical type of intervention, inadequate allocation concealment, and nontop 5 medical journals were less likely to report MPD. We also conducted a linear regression with "the percentage of MPD" as dependent variable and the same independent variables described previously and the same directional hypotheses.

\subsubsection{Planning and conduct of analyses addressing $M P D$}

We documented the frequency of planned analysis regarding MPD for all continuous outcomes and the analysis conducted by trial investigators for the chosen outcome. We planned to conduct a logistic regression with whether trials planned a sensitivity analysis regarding MPD as the dependant variable and the independent variables as noted previously. Our a priori hypotheses were as follows: trials with smaller sample size, for-profit type of funding, nonpharmaceutical type of intervention, inadequate allocation concealment, and nontop 5 medical journals would be less likely to plan a sensitivity analysis regarding MPD.

The analysis was performed using the SPSS software, version 22/12 (IBM Corp., TX, USA).

\section{Results}

\subsection{General characteristics of included RCTs}

We included 200 eligible trials that met out target sample size (Fig. 1). Agreement between reviewers was substantial: kappa of 0.63 for title and abstract screening and 0.64 for full-text screening.

Table 1 presents the general trial characteristics and Table 2 the methodological characteristics of the eligible studies. Symptoms $(84,42 \%)$, quality of life $(44,22 \%)$, and functional status $(33,16.5 \%)$ were the most frequently investigated continuous patient-important outcomes. All but one trial reported time at which patients were followed up; the median follow-up time was 3.3 months (interquartile range of 0.7-12 months). Of these 199 trials, 92 (46\%) reported a single follow-up time and 107 (53.5\%) multiple follow-up times.

\subsection{Reporting and extent of MPD}

Table 3 presents information regarding the reporting of missing participant data. Among the 200 trials, 187 $(93.5 \%)$ had, in the main text or CONSORT flow diagram, an explicit statement of whether MPD occurred. Among the 187 trials that explicitly reported the presence or absence of MPD, 24 (12\%) explicitly stated MPD did not occur, and $163(81.5 \%)$ explicitly reported the extent of MPD, of which $44(27 \%)$ trials reported the percentage of MPD in each arm and overall; the overall median and interquartile range of participants in all time points with MPD were $11.4 \%(2.5-22.6 \%)$. The reporting of MPD was mainly focused on the number of patients who had MPD for the overall study sample but not by the specific outcome.

For 91 trials that included multiple follow-up times and reported MPD from either overall or per arm or both, the median and interquartile range for the percentage of total missing data points were $13.1 \%(6.1-23.7 \%)$. At the last follow-up time, the median and interquartile range for the percentage of missing data points were $14.4 \%$ (7.4-23.6\%). None of the differences between intervention and control in the frequency of missing data approached conventional levels of statistical significance.

We could not conduct the logistic regression with the dependent variable explicitly reporting (or not) the occurrence of MPD because of the small number of studies $(13,6.5 \%)$ that failed to report whether MPD occurred.

We conducted a multiple linear regression addressing the percentage of participants with MPD based on sample size, type of intervention, funding, journal, and allocation concealment. A significant beta coefficient indicated that there was a higher percentage MPD when sample size was larger (beta coefficient $0.01[0.0-0.02], P=0.005$, meaning the MPD would be $1 \%$ more for each 100 patients), with an $\mathrm{R}^{2}$ of 0.17 (Appendix $\mathrm{C}$ at www.jclinepi. com). We further explored the correlation between larger sample size and higher percentage of missing data using a bivariate analysis and found a correlation coefficient of 

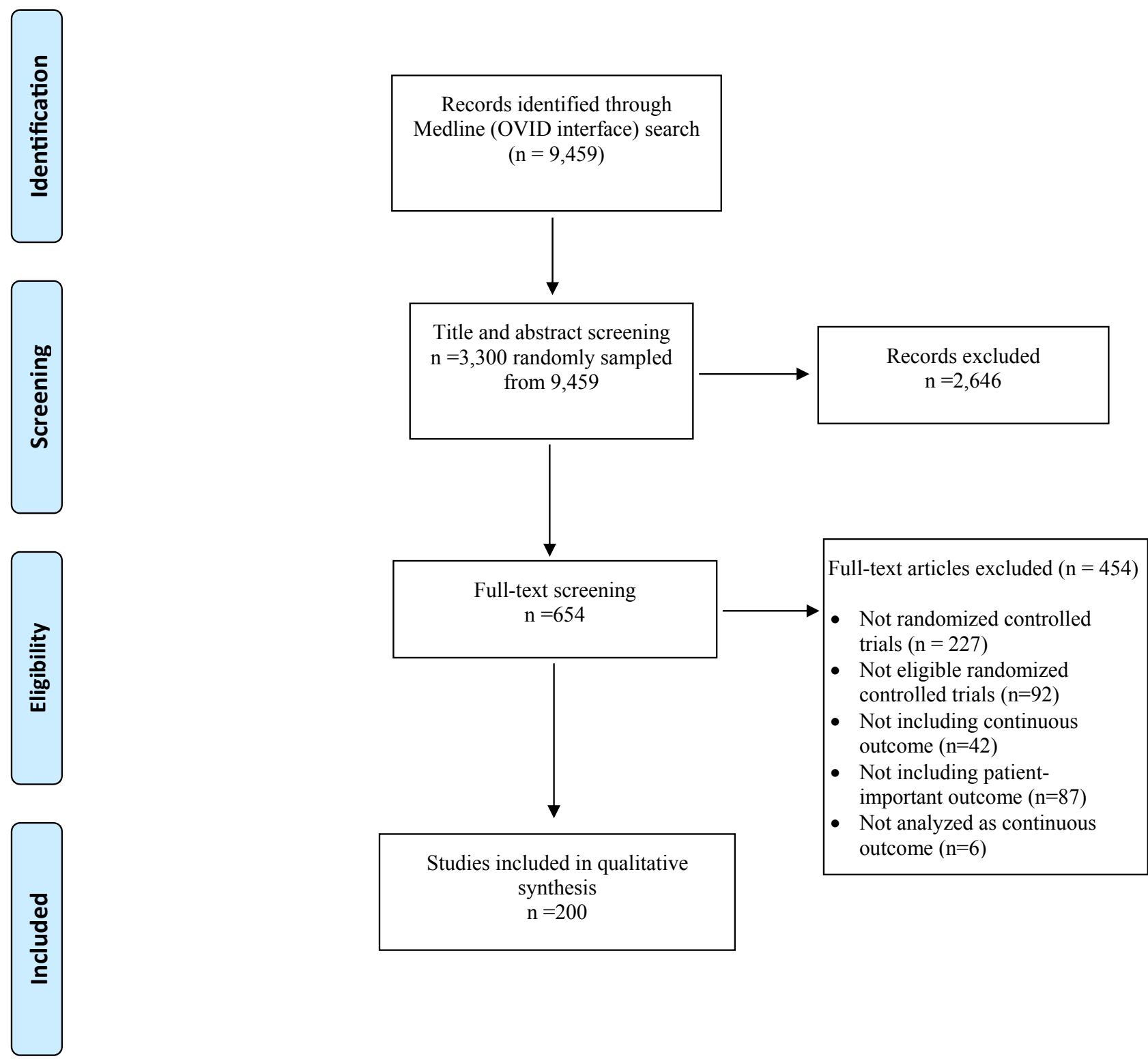

Fig. 1. PRISMA flow diagram.

$0.29(P=0.001)$. Another significant beta coefficient indicated that there was a lower percentage of MPD when funding was not explicitly reported (beta coefficient 4.89 [0.40-9.38], $P=0.03$ ). Type of intervention, journal, and allocation concealment proved not to be significant predictors for the percentage of MPD.

\subsection{Categories of trial participants trial investigators considered as having MPD}

Appendix D at www.jclinepi.com provides data regarding studies' reports of the reasons for MPD. Of 200 included studies, 24 explicitly reported absence of MPD, the remaining 176 studies potentially had MPD. The most frequently considered categories for potential MPD were "withdrew consent" (81 trials, 46.0\%) and "experienced adverse event" (41 studies, 23.3\%).

\subsection{Analyses reported in the methods section regarding $M P D$}

Table 4 presents the analysis plan reported in the methods section of included trials. Among all 200 included studies, 58 (29\%) and 21 (10.5\%) reported, in the methods section of their article, a plan to handle MPD in their primary and sensitivity analysis, respectively. The most frequent approaches specified were last observation carried forward (LOCF) $(11,5.5 \%)$ and mixed-effect model (11, $5.5 \%)$ for the primary analysis, and multiple imputation (MI) for the sensitivity analysis. 
Table 1. General characteristics of 200 included trials to determine effect of missing participant data on outcomes

\begin{tabular}{|c|c|}
\hline Variable & $n(\%)$ \\
\hline \multicolumn{2}{|l|}{ Outcome classification } \\
\hline Efficacy & $191(95.5)$ \\
\hline Safety & $9(4.5)$ \\
\hline \multicolumn{2}{|l|}{ Types of chosen outcome } \\
\hline Length of stay (in hospital, intensive care unit) & $25(12.5)$ \\
\hline Symptoms & $84(42.0)$ \\
\hline Quality of life & $44(22.0)$ \\
\hline Functional status & $33(16.5)$ \\
\hline Disease severity & $8(4.0)$ \\
\hline Length of drug use & $6(3.0)$ \\
\hline \multicolumn{2}{|l|}{ Intervention } \\
\hline Pharmacological & $86(43.0)$ \\
\hline Surgical & $24(12.0)$ \\
\hline Invasive nonsurgical procedure & $14(7.0)$ \\
\hline Rehabilitation & $24(12.0)$ \\
\hline Behavioral intervention & $24(12.0)$ \\
\hline Diagnostic test & $1(0.5)$ \\
\hline Complementary and alternative medicine & $3(1.5)$ \\
\hline Other & $24(12.0)$ \\
\hline \multicolumn{2}{|l|}{ Control } \\
\hline Standard care & $47(23.5)$ \\
\hline Placebo/sham & $61(30.5)$ \\
\hline Pharmacological & $31(15.5)$ \\
\hline Surgical & $16(8.0)$ \\
\hline Invasive nonsurgical procedure & $7(3.5)$ \\
\hline Rehabilitation & $12(6.0)$ \\
\hline Behavioral intervention & $10(5.0)$ \\
\hline Diagnostic test & $1(0.5)$ \\
\hline \multicolumn{2}{|l|}{ Number of centers } \\
\hline Single center & $102(51.0)$ \\
\hline Multicenters & $98(49.0)$ \\
\hline \multicolumn{2}{|l|}{ Journal types } \\
\hline Top 5 journals & $37(18.5)$ \\
\hline Nontop 5 journals & $163(81.5)$ \\
\hline \multicolumn{2}{|l|}{ Arms } \\
\hline 2 arms & $154(77.0)$ \\
\hline More than 2 arms & $46(33.0)$ \\
\hline \multicolumn{2}{|l|}{ Funding ${ }^{\mathrm{a}}$} \\
\hline Private for profit (only provide drugs) & $35(17.5)$ \\
\hline Private for profit (provide things other than drugs) & $38(19.0)$ \\
\hline Private not for profit & $72(36.0)$ \\
\hline Governmental & $78(39.0)$ \\
\hline Not funded & $13(6.5)$ \\
\hline Not reported & $25(12.5)$ \\
\hline
\end{tabular}

${ }^{a}$ Adds up to more than 200 because some trials have more than one source of funding.

\subsection{Analyses conducted regarding MPD}

Table 5 presents the analysis approaches authors used regarding MPD. Among 163 trials explicitly reporting the occurrence of MPD, it was unclear how trialists dealt with MPD in their primary analysis in $16(9.8 \%)$ trials. Of the remaining 147 trials, 74 (45.4\%) used complete case analysis, $35(21.5 \%)$ all available data analysis, 9 (5.5\%) LOCF, $10(6.1 \%)$ mixed-effect model, 9 (5.5\%) MI, 3 (1.8\%) maximum likelihood, 2 (1.2\%) mean imputation, 1 $(0.6 \%)$ regression, and $4(2.5 \%)$ methods other than the aforementioned. Very few $(14,8.6 \%)$ trials specified the missing mechanism when conducting an analysis regarding
Table 2. Methodological characteristics of 200 included trials to determine effect of missing participant data on outcomes

\begin{tabular}{lr}
\hline Variable & $\boldsymbol{n}(\%)$ \\
\hline Allocation concealment ${ }^{\mathrm{a}}$ & $139(69.5)$ \\
$\quad$ Adequate & $61(30.5)$ \\
$\quad$ Inadequate & \\
Blinding $^{\mathrm{b}}$ & $99(49.5)$ \\
$\quad$ Patients & $80(40.0)$ \\
$\quad$ Providers & $106(53.0)$ \\
$\quad$ Data collectors & $105(52.5)$ \\
$\quad$ Outcome adjudicators & $21(10.5)$ \\
$\quad$ Data analysts & $198(99.0)^{c}$ \\
No early stopping for benefit & $5(2.5)$ \\
Primary analysis authors described & $94(47.0)$ \\
$\quad$ Analysis described as intention to treat & $11(5.5)$ \\
$\quad$ Analysis described as modified intention to treat & \\
Analyzed participants for whom outcome data were & \\
$\quad$ available in group to which they were randomized & \\
$\quad$ No explicit statement & $63(31.5)$ \\
Per protocol analysis & $27(13.5)$ \\
\hline
\end{tabular}

a Allocation concealment refers to judgment of "definitely concealed" or "probably concealed."

b Blinding refers to judgment of "definitely blinded" or "probably blinded."

c 198 (99\%) trials did not stop the investigation early for benefit.

MPD; in 13 of 14 that did make such an explicit statement, the assumption was MAR (Table 4).

Of these 163 studies, $16(9.8 \%)$ studies conducted sensitivity analysis for MPD (more than one sensitivity analysis can be conducted); multiple imputation $(4,2.5 \%)$, complete case analysis $(3,1.8 \%)$, LOCF $(2,1.2 \%)$, mean imputation $(1,0.6 \%)$, combination of more than one method $(1$, $0.6 \%)$, other methods not mentioned previously $(2,1.2 \%)$, and not reported $(6,4.5 \%)$. Five studies reported missing data assumptions used for the sensitivity analysis, and all of them used the same assumption as their primary analysis. The remaining 11 studies did not report whether they changed underlying missing data assumptions. Of the 16 trials that reported results of a sensitivity analyses to assess the impact of MPD, two reported that results were no longer statistically significant in one of their sensitivity analyses. Of 163 trials reporting the occurrence of MPD, 18 (11.1\%) discussed the implications of MPD regarding risk of bias.

We could not perform the planned logistic regression to explore the factors associated with whether trials planned sensitivity analysis regarding MPD, due to the small number of studies conducting sensitivity analysis.

\section{Discussion}

\subsection{Key findings}

Of 200 RCTs, 187 (93.5\%) made explicit statements regarding MPD, of which 24 (12\%) reported no MPD and $163(81.5 \%)$ reported that MPD had occurred, and its extent (Table 2). Very few investigators (16, 9.8\%) compared baseline characteristics between patients with 
Table 3. Reporting of information regarding missing participant data in included trials to determine effect of missing participant data on outcomes

\begin{tabular}{lc}
\hline Variable & $\boldsymbol{n}(\%)$ \\
\hline Among all included studies $(n=200)$ & \\
$\quad$ Explicit statement about missing participant data occurred in the \\
$\quad$ main text or CONSORT flow diagram & \\
$\quad$ Yes, stated MPD occurred & $163(81.5)$ \\
$\quad$ Yes, stated MPD did not occur & $24(12.0)$ \\
$\quad$ No explicit statement & $13(6.5)$ \\
Among studies reported MPD occurred ( $n=163)$ & \\
Assessment of baseline characteristics & \\
Yes, MPD group vs. non-MPD group & $12(7.4)$ \\
$\quad$ Yes, MPD in 1st arm vs. MPD in 2nd arm & $2(1.2)$ \\
$\quad$ Yes, they did both that mentioned previously & $1(0.61)$ \\
$\quad$ No & $148(90.8)$ \\
Reporting of MPD & \\
Separately reported for two arms & $114(69.9)$ \\
Reported overall only & $5(3.1)$ \\
Reported both per arm and overall & $44(27.0)$ \\
\hline
\end{tabular}

Abbreviation: MPD, missing participant data.

missing data and patients with complete data (Table 3). Many of these 163 trials reported substantial MPD (median 11.4\%, Q1 2.5\% to Q3 22.6\%).

Among all 200 trials, less than a third (58 trials, 29\%) reported in their methods a planned analytical approach to address MPD in their primary analysis and even fewer

Table 4. Method section reported analytic approach in 200 included trials for MPD on any continuous outcome

\begin{tabular}{lr}
\hline Variable & $\boldsymbol{n}(\%)$ \\
\hline Primary analysis & \\
Yes, reported in methods & $58(29.0)$ \\
$\quad$ No, did not report in the methods & $142(71)$ \\
What primary analysis was reported in methods & \\
$\quad$ ( $=58)$ & \\
Complete case analysis & $9(4.5)$ \\
All available data analyses & $3(1.5)$ \\
Mean imputation & $3(1.5)$ \\
Last observation carrying forward (LOCF) & $11(5.5)$ \\
Regression for MPD & $1(0.5)$ \\
Multiple imputation (MI) & $9(4.5)$ \\
Maximum likelihood (ML) & $3(1.5)$ \\
Mixed-effect model for missing data & $11(5.5)$ \\
Other & $6(3.0)$ \\
Not reported & $2(0.0)$ \\
Sensitivity analysis & \\
Yes, reported in methods & $21(10.5)$ \\
No, did not report in the methods & $179(89.5)$ \\
What sensitivity analysis was reported in methods & \\
$\quad$ (N = 21) & \\
Complete case analysis & $3(1.5)$ \\
Mean imputation & $1(0.5)$ \\
Last observation carrying forward (LOCF) & $2(1.0)$ \\
Regression for MPD & $1(0.5)$ \\
Multiple imputation (MI) & $8(4.0)$ \\
Mixed-effect model for missing data & $1(0.5)$ \\
Other & $3(1.5)$ \\
Not reported & $2(1.0)$ \\
\hline
\end{tabular}

Abbreviation: MPD, missing participant data.
Table 5. Reporting of information regarding analysis of missing participant data in included trials on patient-important outcomes

\begin{tabular}{|c|c|}
\hline Variable & $n(\%)$ \\
\hline \multicolumn{2}{|l|}{ Among studies reported MPD occurred $(N=163)$} \\
\hline \multicolumn{2}{|l|}{ Assumed missing mechanism when conduct analysis } \\
\hline Missing at random & $13(7.9)$ \\
\hline Ignorable missing & $1(0.7)$ \\
\hline Not stated & $149(91.4)$ \\
\hline \multicolumn{2}{|l|}{ Primary analysis } \\
\hline Complete case analysis & $74(45.5)$ \\
\hline All available data analyses & $35(21.5)$ \\
\hline Mean imputation & $2(1.2)$ \\
\hline Last observation carrying forward (LOCF) & $9(5.5)$ \\
\hline Regression for MPD & $1(0.6)$ \\
\hline Multiple imputation (MI) & $9(5.5)$ \\
\hline Maximum likelihood (ML) & $3(1.8)$ \\
\hline Mixed-effect model for missing data & $10(6.1)$ \\
\hline Other & $4(2.5)$ \\
\hline Unclear & $16(9.8)$ \\
\hline $\begin{array}{l}\text { Provide justification for the method used to handle } \\
\text { MPD in the primary analysis }\end{array}$ & $9(5.5)$ \\
\hline \multicolumn{2}{|c|}{$\begin{array}{l}\text { Whether they conducted sensitivity analysis regarding MPD for } \\
\text { chosen outcome }\end{array}$} \\
\hline Yes & $16(9.8)$ \\
\hline No & $147(90.2)$ \\
\hline $\begin{array}{l}\text { Implications of MPD regarding risk of bias } \\
\text { discussed }\end{array}$ & $18(11.0)$ \\
\hline \multicolumn{2}{|l|}{$\begin{array}{l}\text { Among studies conducted sensitivity analysis regarding MPD } \\
\qquad(N=16)^{a}\end{array}$} \\
\hline Complete case analysis & $3(1.8)$ \\
\hline Mean imputation & $1(0.6)$ \\
\hline Last observation carrying forward (LOCF) & $2(1.2)$ \\
\hline Multiple imputation (MI) & $4(2.5)$ \\
\hline Combination of more than one method above for MPD & $1(0.6)$ \\
\hline Other & $2(1.2)$ \\
\hline Not reported & $6(4.5)$ \\
\hline $\begin{array}{l}\text { Sensitivity analysis changed the statistical significant } \\
\text { result }\end{array}$ & $2(1.2)$ \\
\hline
\end{tabular}

Abbreviation: MPD, missing participant data.

a $N=16$; it is possible that there are more than one sensitivity analysis.

(21 trials, 10.5\%) reported a plan for a sensitivity analysis (Table 4). Very few $(14,8.6 \%)$ trials specified the missing mechanism when conducting an analysis regarding MPD; in 13 of 14 that did make an explicit statement, the assumption was MAR (Table 5). The most common way trialists handled MPD was a complete case or all available data analysis $(109,67 \%)$. Other approaches included mixedeffect models $(10,6.1 \%)$, LOCF $(9,4.5 \%)$, and MI (9, $4.5 \%)$. Of the 163 trials with MPD, $16(9.8 \%)$ conducted a sensitivity analysis.

\subsection{Strengths and limitations of study}

Strengths of our study include a systematic and comprehensive search, independent and duplicate screening and data abstraction, and a focus on patient-important continuous outcomes. We also implemented standardized built-in instructions in both screening and data abstraction forms on the Web-based systematic review software and 
conducted calibration exercises. Our random sample of eligible studies from the 119 core medical journals published in 2014 ensures high representativeness of the recent practice among trialists [23-25].

With regard to limitations, we captured only the information authors reported in the publication and in the additional information provided in the appendix and supplementary data files. Authors may have conducted analyses regarding MPD beyond what they reported - contact with authors, which we did not undertake, could have provided additional information in this regard. We could also have checked registered protocols of trials for their MPD analyses plan when applicable. We did not collect data regarding whether the continuous outcomes selected are single or repeated measured outcomes. Collecting such information might provide more details on the different approaches of analyzing MPD on these two types of continuous outcomes. Finally, we could have adjusted for potential clustering effect for papers published in the same journal since they might have followed the same requirement from the journal to report the article in a certain manner.

\subsection{Relation to other studies}

Akl et al. [12] investigated the extent of MPD, the reporting, and the impact on results associated with MPD in studies addressing binary outcomes in five general prestigious medical journals. They found $13 \%$ of the trials did not report whether MPD occurred and 20\% did not clearly report the analytical approaches used to handle MPD. These results are very similar to what we found with respect to continuous outcomes. Alshurafa et al. [26] investigated how methodological articles defined intention to treat (ITT) analysis in the context of MPD. They found the most frequently mentioned strategies suggested to deal with MPD within ITT were LOCF (50\%), sensitivity analysis (50\%), and imputation (46\%). We found investigators took advantage (though infrequently) of a wider variety of sophisticated statistical strategies, and did not frequently use LOCF. Fiero et al. [27] conducted a systematic survey on how cluster RCTs dealing with MPD for their primary analysis. They found 19\% of participants have missing data and the most common method to handle MPD is complete case analysis (44, $55 \%$ ). Bell et al. [28] conducted a review of how RCTs handle missing data in the BMJ, JAMA, Lancet, and New England Journal of Medicine excluding cluster randomized trials and trials with primary outcome as survival data. Among 77 included trials, the median percentage of participants with missing outcome was 9\% (range 0-70\%) and 27 (35\%) trials with missing data reported a sensitivity analysis. Existing review on methods regarding binary outcomes, cluster RCTs and top medical journals appears to be consistent with what we found for continuous outcomes in RCTs.

\subsection{Interpretation of findings}

In a recently conducted systematic survey [29] we conducted on the performance of methods of handling continuous MPD, LOCF proved to be much worse, particularly with respect to bias, than other methods investigated. In our study, investigators seem aware of the limitations of LOCF, with only 9 of 200 studies using the method, possibly much less than in the past. On the other hand, mixed-effects models or multiple imputation, approaches that proved to have excellent properties in the studies summarized in the survey, were used no more frequently than LOCF.

We found an association between explicit reporting of MPD with explicit reporting of funding. This suggests an association between not reporting MPD and poor reporting of other trial aspects. We also found trials with larger sample size had larger percentages of missing data. This finding highlights both the challenges of minimizing MPD in larger trials and enhances the importance of planning optimal analytical strategies to handle potential MPD.

\subsection{Implications for trialists}

Trial investigators should be more explicit in providing details on the reporting of MPD both at participant level and at outcome level, particularly when trials have multiple follow-up times as is commonly seen in the context of continuous outcomes. Reporting only the number of patients missing without specification of MPD at outcome level may omit key information.

Ideally, investigators will institute measures to minimize MPD [30]. MPD is often, however, inevitable, and implementation of optimal analytic strategies to deal with MPD would be highly desirable. These strategies include developing in advance a plan to deal with MPD and reporting that plan in their protocol and ultimately in the methods section of articles. Investigators should determine if baseline characteristics and other covariates differ between patients with missing data and patients with complete data. Differences in characteristics suggest that data might be MAR or that they could even be NMAR. Furthermore, they should examine the relation between patients' characteristics and observed outcomes; if there are substantial associations, it also suggests that, to some extent, the data are MAR and that imputation and data augmentation methods may be useful, at least as sensitivity analyses. Because it is very likely that in most cases both MAR and NMAR mechanisms are at play, sensitivity analysis testing assumptions about missing data should also be a standard of practice.

Investigators should be aware of the current optimal methods for handling MPD such as mixed-effect models and avoid using poorer performing methods such as LOCF or other single imputation methods [29]. The use of more sophisticated methods is likely to require help from 
statisticians. Investigators should provide a justification for the sensitivity analyses they choose and discuss the implication of sensitivity analyses of MPD regarding risk of bias.

\subsection{Implications for systematic reviewers}

When judging the risk of bias of included trials, systematic reviewers should examine the quality and extent of reporting MPD in CONSORT and text of the trials at an outcome of interest level. Furthermore, they should examine the sensitivity analysis regarding MPD conducted in individual trials; this may provide a sense of the extent of risk of bias related to MPD across studies. These results may influence the application of across-trial methods to estimate the impact of MPD on risk of bias across the body of evidence [31,32].

\subsection{Implications for future research}

A checklist addressing the reporting of analysis regarding MPD in RCTs may be useful for both evaluating and optimizing analytic strategies in studies of continuous outcomes. Further investigation might focus on optimal approaches to conducting sensitivity analysis regarding MPD.

\section{Acknowledgments}

The authors thank Lawrence Mbuagbaw for the assistance with data analyses. The authors also thank Rachel Couban for her assistance with reference uploading with DistillerSR.

Authors' contributions: Y.Z., E.A.A., L.T., and G.G. were responsible for study conception and design. Y.Z., I.D.F., L.C.L., S.A.K., F.A.B.A., A.L., S.C., L.C.L., T.D., W.W., J.J.R., S.Z., A.A., M.W., X.J., G.P.M., Y.Z., A.C.-L., P.A., Y.F., and L.A.K. screened and acquired the data. Y.Z. analyzed the data. Y.Z. drafted the article and is a guarantor. All authors critically revised the article and approved the final version.

\section{Supplementary Data}

Supplementary data related to this article can be found at http://dx.doi.org/10.1016/j.jclinepi.2017.05.017.

\section{References}

[1] Akl EA, Kahale LA, Agarwal A, Al-Matari N, Ebrahim S, Alexander PE, et al. Impact of missing participant data for dichotomous outcomes on pooled effect estimates in systematic reviews: a protocol for a methodological study. Syst Rev 2014;3:137.

[2] McKnight PE, MK SS, Figueredo AJ. Missing data: a gentle introduction. New York, NY: Guilford; 2007.

[3] te Riele WW, Boerma D, Wiezer MJ, Borel Rinkes IH, van Ramshorst B. Long-term results of laparoscopic adjustable gastric banding in patients lost to follow-up. Br J Surg 2010;97:1535-40.
[4] Geng EH, Emenyonu N, Bwana MB, Glidden DV, Martin JN. Sampling-based approach to determining outcomes of patients lost to follow-up in antiretroviral therapy scale-up programs in Africa. JAMA 2008;300:506-7.

[5] Montori VM, Guyatt GH. Intention-to-treat principle. CMAJ 2001; 165:1339-41.

[6] Little RJ, Rubin D. Statistical analysis with missing data. New York: Wiley; 2002.

[7] Dziura JD, Post LA, Zhao Q, Fu Z, Peduzzi P. Strategies for dealing with missing data in clinical trials: from design to analysis. Yale J Biol Med 2013;86:343-58.

[8] Sterne JA, White IR, Carlin JB, Spratt M, Royston P, Kenward MG, et al. Multiple imputation for missing data in epidemiological and clinical research: potential and pitfalls. BMJ 2009; 338:b2393.

[9] Wood AM, White IR, Thompson SG. Are missing outcome data adequately handled? A review of published randomized controlled trials in major medical journals. Clin Trials 2004;1:368-76.

[10] Hollis S, Campbell F. What is meant by intention to treat analysis? Survey of published randomised controlled trials. BMJ 1999;319: 670-4.

[11] Baron G, Boutron I, Giraudeau B, Ravaud P. Violation of the intentto-treat principle and rate of missing data in superiority trials assessing structural outcomes in rheumatic diseases. Arthritis Rheum 2005; 52:1858-65.

[12] Akl EA, Briel M, You JJ, Sun X, Johnston BC, Busse JW, et al. Potential impact on estimated treatment effects of information lost to follow-up in randomised controlled trials (LOST-IT): systematic review. BMJ 2012;344:e2809.

[13] Yi GY, He W. Median regression models for longitudinal data with dropouts. Biometrics 2009;65:618-25.

[14] Thabane L, Mbuagbaw L, Zhang S, Samaan Z, Marcucci M, Ye C, et al. A tutorial on sensitivity analyses in clinical trials: the what, why, when and how. BMC Med Res Methodol 2013;13:92.

[15] Horvitz-Lennon M, James O'Malley A, Frank RG, Normand S-LT. Improving traditional intention-to-treat analyses: a new approach. Psychol Med 2005;35:961-70.

[16] Huang X, Jiao L, Wei L, Quan H, Teoh L, Koch GG. Missing radiographic data handling in randomized clinical trials in rheumatoid arthritis. J Biopharm Stat 2013;23:1435-52.

[17] Li X, Wang WW, Liu GF, Chan IS. Handling missing data in vaccine clinical trials for immunogenicity and safety evaluation. J Biopharm Stat 2011;21:294-310.

[18] Liu M, Wei L, Zhang J. Review of guidelines and literature for handling missing data in longitudinal clinical trials with a case study. Pharm Stat 2006;5:7-18.

[19] Tsonaka R, Verbeke G, Lesaffre E. A semi-parametric shared parameter model to handle nonmonotone nonignorable missingness. Biometrics 2009;65:81-7.

[20] Unnebrink K, Windeler J. Intention-to-treat: methods for dealing with missing values in clinical trials of progressively deteriorating diseases. Stat Med 2001;20:3931-46.

[21] Allison PD. Missing data. Thousand Oaks,CA: Sage; 2002.

[22] Shea BJ, Grimshaw JM, Wells GA, Boers M, Andersson N, Hamel C, et al. Development of AMSTAR: a measurement tool to assess the methodological quality of systematic reviews. BMC Med Res Methodol 2007;7:10.

[23] Sun X, Briel M, Busse JW, You JJ, Akl EA, Mejza F, et al. The influence of study characteristics on reporting of subgroup analyses in randomised controlled trials: systematic review. BMJ 2011;342: d1569.

[24] Montori VM, Devereaux PJ, Adhikari NK, Burns KE, Eggert CH, Briel M, et al. Randomized trials stopped early for benefit: a systematic review. JAMA 2005;294:2203-9.

[25] Lee KP, Schotland M, Bacchetti P, Bero LA. Association of journal quality indicators with methodological quality of clinical research articles. JAMA 2002;287:2805-8. 
[26] Alshurafa M, Briel M, Akl EA, Haines T, Moayyedi P, Gentles SJ, et al. Inconsistent definitions for intention-to-treat in relation to missing outcome data: systematic review of the methods literature. PLoS One 2012;7:e49163.

[27] Fiero MH, Huang S, Oren E, Bell ML. Statistical analysis and handling of missing data in cluster randomized trials: a systematic review. Trials 2016;17:72.

[28] Bell ML, Fiero M, Horton NJ, Hsu CH. Handling missing data in RCTs; a review of the top medical journals. BMC Med Res Methodol 2014; 14:118.

[29] Zhang Y, Alyass A, Vanniyasingam T, Sadeghirad B, Flórez ID, Pichika SC, et al. A systematic survey of the methods literature on the reporting quality and optimal methods of handling participants with missing outcome data for continuous outcomes in randomized controlled trials. J Clin Epidemiol. 2017 Jun 1. pii: S08954356(17)30572-3.

[30] Scharfstein DO, Hogan J, Herman A. On the prevention and analysis of missing data in randomized clinical trials: the state of the art. J Bone Joint Surg Am 2012;94:80-4.

[31] Ebrahim S, Johnston BC, Akl EA, Mustafa RA, Sun X, Walter SD, et al. Addressing continuous data measured with different instruments for participants excluded from trial analysis: a guide for systematic reviewers. J Clin Epidemiol 2014;67: $560-70$.

[32] Ebrahim S, Akl EA, Mustafa RA, Sun X, Walter SD, HeelsAnsdell D, et al. Addressing continuous data for participants excluded from trial analysis: a guide for systematic reviewers. J Clin Epidemiol 2013;66:1014-1021.e1. 